Review of:

\title{
Small-strain shear stiffness of compacted bentonites for engineered barrier system
}

The emphasis of the paper is on the small strain shear stiffness of compacted bentonites in light of their use in engineered barrier systems for deep geological disposal.

In its current form the paper has some deficiencies that need to be addressed before it can be considered for publication.

The primary issues with the paper can be summarized as follows:

- the motivation for the work in not well defined. Specifically, a poor case is made in the inteduction for studying the small strain stiffness of compacted bentonites. We $\overline{\overline{\text { arre }}}$ told that the nature of bentonite makes study of its mechanical behavior difficult (I do not find this statement well founded), and that because of this there are few data available in the literature. This in itself is not a good reason to pursue any investigation.

- the authors are not effective in providing context for their work: the review of previous wor $\equiv$ ovided in the introduction is rather scattered, with no

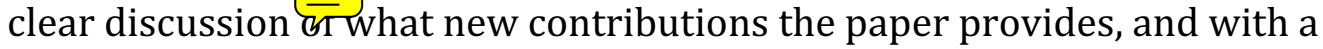
questionable choice of the previous research covered.

- the lessons from the work are also not well conveyed to the reader. No hypothesis is provida for some of the observations drawn from the data, for example the observea change in the stress level exponent with degree of saturation, or the presence of a G0 maximum at an intermediate value of S.

- the discussion of some of the fitting/modelling efforts appear acritical, and the last part of the paper (on the application of the model by Alonso) is rushed with makey pieces left unexplained.

- the paper requires improvements in the English form and the organization.

- several figures are presented with no discussion at all.

- many references annear poorly chosen, and in some cases appear irrelevant to the work preser $\overline{\overline{\bar{\nu}}}$.

Note: if resubmitted, the paper should include numbered lines to facilitate the reviewing p层ss.

More detailed comments for the various sections of the paper are provided below.

\section{INTRODUCTION}

The significance of the small strain behavior of compacted bentonites in the context of their use in EBS is not well conveyed.

Beyond this, the rest of the introduction is disconnected and poorly thought out. We are given some data on the two materials examined and some information on how their behavior has been modeled (why here?). This information would likely 
better belong in a materials section, or even later in the paper when analyzing the results (e.g. what is $\equiv$ point for the reader now to be told that the water retention curve of the FEBEX bentonite can be modeled using a particular model?). The authors also provide information on the in situ placement conditions, although from the text it is actually unclear whether this has already taken place.

The rest of the introduction focuses on previous work, but the criterion for selecting these previous results is unclear, as is the connection to the research presented in the paper. We are told, for exapale, that studies have been performed on the analysis of shearing in canisters ( about what these studies found (why bother mentioning them?), or how their results relate to the work presented.

The subsequent text on measurement of soil properties, with rather general references on the combined use of triaxial and resonant column testing to provide stiffness over a broad range of $\equiv$ ins, and a list of studies on the small strain behavior of compacted soils, als brings very little to the paper. What do these studies find or do that is relevant to the work presented? What is the connection between the study performed and, for example, the referenced work on rock-block and block-block interfaces?

Finally, the discussion of the models describing the variation of the small strain shear stiffness parameters (probably of most interest in the context of the work performed by the authors) is suppuficial and not well thought out: why reference expressions for cohesionless soils $\overline{\bar{r}}$ when so much work has been conducted on clays? And why not provide more details on the models presented for unsaturated conditions?

In conclusion, this section falls short in both convincing the authors of the significance of the work presented, and in describing the context in which the work fits. This section requires major revisions.

\section{EXPERIMENTAL METHODS AND RESULTS}

This section should be separated in two distinct sections, one on experimental methods (probably including some of the material information currently included in the introduction) and one on results.

Clarify testing program, and specifically different series of tests presented in tables 2 and 3.

What is the "rebound effect" that supposedly makes control of dry density difficult in compacted so

What are the "buffer blocks" referred to in this section? The subsequent statement on the different changes is also uncrear. 
The authors state that the confining stress was applied using compressed air. This is, in general, and undesirable $\mathrm{cl} \equiv$, especially in tests aimed at illustrating the role of degree of saturation.

Figure 6 presents data of vertical strain versus isotropic confinement for MX-80 specimens. Why are only selecte $\equiv$ ta included? What was selection criterion? What is the expression in Equation (4) useful for?

The discussion of Figure 7 is unclear. The authors should start by clearly explaining what Figure 7 shows and what the relationship of beta versus dry density signifies. The statement that the higher values of beta observed for the HP tests signify the existence of a quasi preconsolidation $\mathrm{p} \equiv$ ure between 400 and $1000 \mathrm{kPa}$ is also confusing. I think Figure 6 shows that clearly. Why not simply derive isotropic yield stress values from Figure 6, instead of making inferences from Figure 7?

Note: I am not able to reconcile some numbers for the HP tests (i.e. derive the values of beta reported in Figure 7 from the strain and stress values shown in Figure 6), i.e. based on Figure 6, I get lower vall $\bar{\equiv}$ of beta than those shown. Can you please check this?

The subsequent derivation of the compressibility parameter kappa, and the long text on the bulk modulus is unneceporily complicated and it is not apparent what the authors want to illustrate. I anvinot sure that all of what is included is really necessary.

Finally, in Figures 8 and 9 the shear stiffness data are presented. It is unclear why the authors feel the need to immediately compare their results to two particular studies (Hoyos et al. and Lo Presti et al.). I am verv familiar with the Lo Presti paper, which presents data for 3 sands and two natulays, i.e. materials that have none or very limited relevance to the work presented in the paper. Similarly, the paper by Hoyos presents data on silty sand. Referencing for the sake of referencing is not a good practice.

Please $\equiv$ e that in this section, the table number is often missing when a table is referenced.

Why not normalize stiffnoss values using available void ration functions, to see if data for one material farivil same band?

Figure 10, 11 and 12 are redundant - basically the same data shown in three different plots (data also included in tables). Focus on what the key take away from these data is, and choose the figure that oc authors have nothing to say about a figure (e.g. Figures 10 and 11), then there is likely no need to include it. 
Figure 12 requires more discussion. The figure suggests different trends in the stiffness of the two bentonites, but no $\overline{\bar{\nu}}$ othesis is provided. I also question whether the contours for the FEBEX bentonite can be considered reliable given the limited data.

\section{INTERPRETATION OF THE RESULTS}

I do not think that Figure 13 is effectiv $\doteq$ conveying that the stress level exponent decreases with increasing degree of saturation, especially for MX-80.

What is the criterion used to establish the linear threshold in Figures 14 and 15? How is this threshold affected by stress level?

The authors' data indicate that the linear threshold is greater for the FEBEX bentonite: are the numbers included in the text correct based on Figure 15?

The authors then go on to analyze the variation of the shear modulus based on a soil model used in Plaxis? What is the purpose of this exercise? What does this tell us? How are we supposed to use this information? Moreover, the scatter in Figure 17 appears huge, especially given the logarithmic scale.

The interpretation of the data in the context of the Alonso model shows some promise but is hard to follow, and some information is missing. For example:

- What is the double structure of bentonites the authors refer to? This requires a brief explanation.

- Why is ns=3 chosen?

- Where does the assumption of linear relationship between em and ew come from?

- How are values of em and betam derived from Figure 18?

- And so on....

I would suggest that a new version of the paper focus on improving this part of the paper, while much of the earlier text/analysis (e.g. discussion of bulk modulus and derivation of beta parameter) could be greatly shortened or eliminated.

I was also hoping the discussion of the results would address some aspects that the previous section had left hanging, in particular

- What is physical mechanism(s) behind observed existence of maximum in shear stiffness?

- What does the change in stress level exponent with degree of saruration tell us?

\section{CONCLUSIONS}


This section requires improvement. In its current form it is quite vague ("The shear moduli are relatively high..."), and key aspects of the data remain unexplained (i.e. why do we see maximum in Go in one clay and not in the other).

The authors indicate that two equations can be used as predictive tools. The first is a widely established expression for G0, the second contains a parameter (see Figure 17) that shows enormous scatter (i.e. unclear how one would pick a value).

Not clear what the "proposed model" refers to, and how one would use it. 